\title{
A NOVEL ALGORITHM TO PREDICT KNEE ANGLE FROM EMG SIGNALS FOR CONTROLLING A LOWER LIMB EXOSKELETON
}

\author{
Inderjeet Singh Dhindsa ${ }^{1}$, Ravinder Agarwal ${ }^{1}$, Hardeep Singh Ryait ${ }^{2}$ \\ ${ }^{1}$ EIED, Thapar University Patiala, Punjab, India \\ ${ }^{2}$ ECE Department, BBSBEC, Fatehgarh Sahib, Punjab, India
}

\begin{abstract}
This paper presents an algorithm for estimation of the intended knee joint angle from sEMG signals acquired from four muscles of upper limb. The algorithm was evaluated by experiments showing the calculated intended motion while performing a simple daily life activity of sitting in a squat position and standing from a squat position. The proposed algorithm uses Mean Absolute Value (MAV) and Root Mean square (RMS) as features and a multi-layer Back Propagation Neural Network (BPN) for predicting the knee angle. Proposed algorithm along with the experimental results are presented. The predicted knee angle can be used to control a lower limb exoskeleton.
\end{abstract}

Keywords: Back propagation, Exoskeleton, feature extraction, sEMG, Neural Network.

Citation: Dhindsa IS, Agarwal R, Ryait HS. A novel algorithm to predict knee angle from EMG signals for controlling a lower limb exoskeleton. CEUR Workshop Proceedings, 2016; 1638: 536-541. DOI: 10.18287/1613-0073-20161638-536-541

\section{Introduction}

Exoskeletons for human performance enhancement are wearable devices and machines that can increase the speed, strength, and endurance of human being. The user provides control signals for the exoskeleton, while the exoskeleton actuators provide majority of the power necessary for performing the task. The human operator applies a scaled down force compared with the load carried by the exoskeleton.

The earliest recorded work in the field of exoskeleton is that of Nicholas Yagn a Russian army engineer. He was granted a US Patent for his work on "Apparatus for facilitating walking, running, and jumping" [1]. Although Yagn's mechanism was designed to augment running, there is no record that the device was ever built and successfully demonstrated. Prominent earliest proposed exoskeletons were General Electric's "HARDIman" ( Human Augmentation Research and Development Investigation) and Los Alamos National Laboratory's"PITMAN"(Powered suit of armour for infantrymen) [3]. 
The development in the field of exoskeleton picked pace in 2001 when the U.S. Defense Advanced Research Projects Agency (DARPA) started a 50 million project named "Exoskeletons for Human Performance Augmentation (EHPA)". The main goal of EHPA was "to increase the capabilities of ground soldiers beyond that of a human". The EHPA program made a number of institutes made research progress in the technologies related to Exoskeletons.

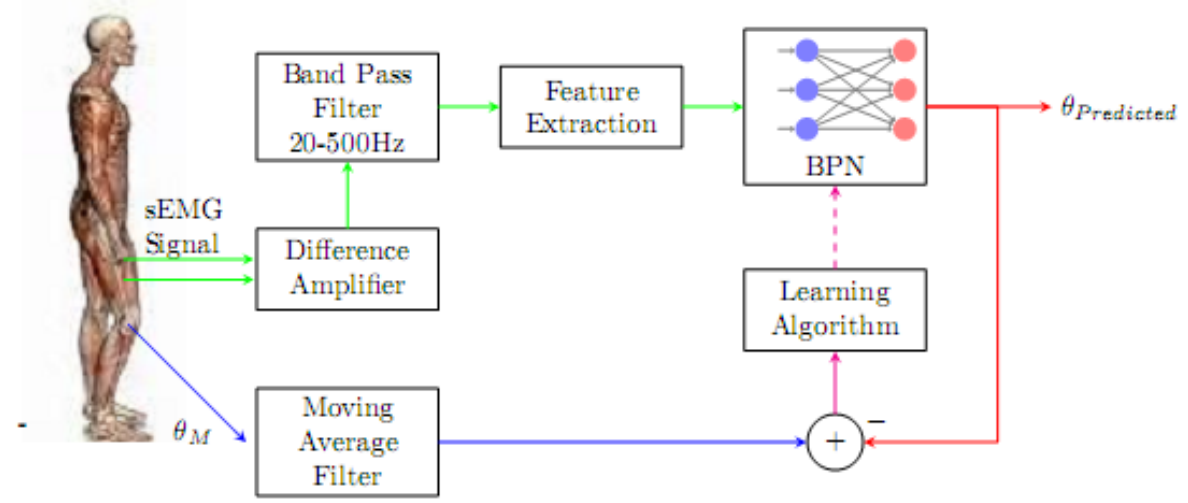

Fig. 1. Block diagram representation of system

Prominent exoskeleton developed under the DARPA project were that of Massachusetts Institute of Technology (MIT) [4], Berkley University's "Berkley Lower Extremity Exoskeleton (BLEEX)" [5] and Sarcos Research Corporation's "Wearable Energetically Autonomous Robot (WEAR)" [6]. All these exoskeletons had one common feature that human motion intent was measured from force sensor placed between the human operator and exoskeleton. The exoskeleton was triggered either by the kinematic position command or by the dynamic contact force command. This introduced a delay in the system. This delay was overcome in the next generation of exoskeleton based on biosensors.

Many of the new proposed exoskeleton predicted the motion intent from EMG signals. Prominent among these exoskeletons was an exoskeleton named Hybrid Assistive Limb (HAL) developed at the University of Tsukuba, Japan, targeted for both performance augmenting and rehabilitative purposes [7]. The main advantage of EMG based exoskeletons is the ability to estimate the forces that will be generated by the muscles before the actual occurrence of mechanical contractions. This information is fed into the exoskeleton system such that by the time the physiological muscles contract the exoskeleton amplifies the joint moment by a preselected gain factor, resulting in a decrease in the reaction time of the human/machine system.

This paper presents an algorithm for estimation of the intended knee joint angle from sEMG signals acquired from four muscles of lower limb namely Vastus Lateralis (VL), Semitendinosus (STEN), Biceps Femoris (BF) and Rectus Femoris (RF). The proposed algorithm uses mean absolute value (MAV) and root mean square (RMS) 
for feature extraction and a multi-layer back propagation neural network (BPN) for predicting the knee angle. The algorithm was evaluated by experiments showing the calculated intended motion while performing a simple daily life activity of sitting in a squat position and standing from a squat position. The algorithm and the experimental results are both presented. The predicted knee angle can be used to control a lower limb exoskeleton.

Table 1. Sensor Position of muscles considered

\begin{tabular}{ccc}
\hline S.no & Muscle & \multicolumn{1}{c}{ Sensor Position } \\
\hline $\mathbf{1}$ & Vastus Lateralis (VL) & $\begin{array}{c}\text { The electrodes need to be placed at } 2 / 3 \text { on } \\
\text { the line from the anterior spina iliaca superior } \\
\text { to the lateral side of the patella } \\
\text { Electrodes need to be placed at } 50 \% \text { on the } \\
\text { line between the ischial tuberosity and the me- } \\
\text { dial epycondyle of the tibia } \\
\text { The electrodes need to be placed at } 50 \% \text { on } \\
\text { the line between the ischial tuberosity and the } \\
\text { lateral epicondyle of the tibia } \\
\text { The electrodes need to be placed at } 50 \% \text { on } \\
\text { the line from the anterior spina iliaca superior } \\
\text { to the superior part of the patella }\end{array}$ \\
\hline & Biceps Femoris (BF) & Rectus Femoris(RF)
\end{tabular}

\section{Methodology}

Five subjects of mean age $31.08 \pm 3.15$ years, mean weight $81.42 \pm 9.5 \mathrm{Kg}$ and mean height $1.72 \pm 0.08 \mathrm{~m}$ respectively, were asked to perform simple daily life activity of sitting in a squat position from standing position and vice versa. sEMG signals were recorded from four muscles of lower limb namely Vastus Lateralis (VL), Semitendinosus (STEN), Biceps Femoris (BF) and Rectus Femoris(RF). EMG signals were recorded using BioTrace+ software for the NeXus-10 biofeedback system at a sampling frequency of $2048 \mathrm{~Hz}$. SENIAM recommendations were followed for the skin preparation and sensor locations $[8,9]$. Reference electrode was placed on the tibia bone. The sensor location for the four muscles are tabulated in Table 1. Block diagram of the joint angle prediction is shown in Fig1. Knee angle was measured using a linear potentiometer based goniometer using NI USB 6009 data acquisition system at a sampling frequency of $2048 \mathrm{~Hz}$. The raw sEMG signal was band pass filtered from $20 \mathrm{~Hz}$ to $500 \mathrm{~Hz}$. The purpose of the lower frequency limit was to remove DC offset and motion artifacts. The high frequency limit prevents the aliasing. This was achieved by using a zero-lag sixth order recursive Butterworth filter. The sEMG signal recorded from VL muscle along with knee angle for subject 1 are plotted in Fig 2. The sEMG signal is not fed directly to a neural network, because of the dimensionality and random characteristics of the signal. Instead features were extracted and fed to the neural network. In this paper, two time domain features mean absolute value (MAV) and root mean square value (RMS) had been used. 


$$
\begin{aligned}
& M A V=\frac{1}{N} \sum_{k=1}^{N}\left|x_{k}\right| \\
& R M S=\sqrt{\frac{1}{N} \sum_{k=1}^{N} x_{k}^{2}}
\end{aligned}
$$

Where $x_{k}$ is thevoltage of $k^{\text {th }}$ sample, $\mathrm{N}$ is number of samples in a segment. The number of samples was set to be 128 for the current study.

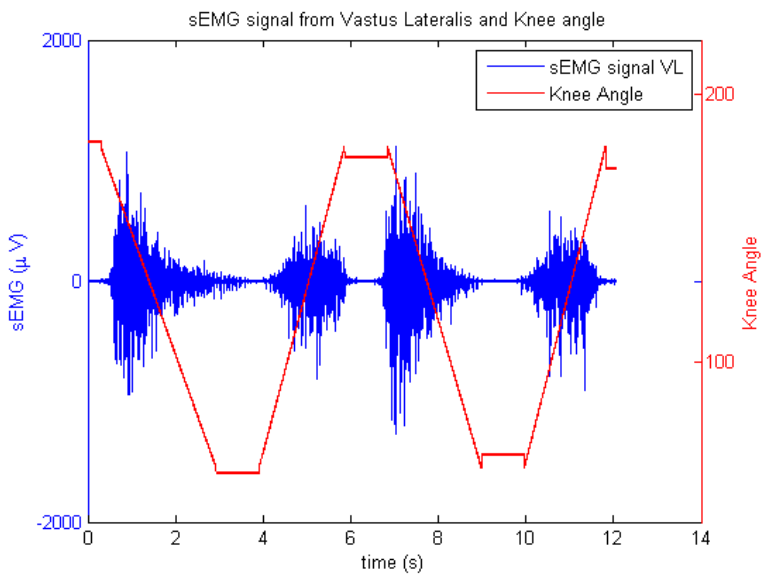

Fig. 2. sEMG signal recorded from Vastus lateralis and measured Knee angle while performing a Stand to Squat to Stand operation

The feature vector is fed to a Back propagation network (BPN). A BPN is used since it learns by example. When Training is finished it returns the output for a particular input. BPN are ideal for simple pattern recognition and mapping tasks. The architecture of the BPN used is shown in Fig 3. The adaptive learning algorithm of the BPN neural network used is explained in following equations:

$$
\begin{aligned}
& E(n)=\frac{1}{2}\left(\theta_{m}-\theta_{a}\right)^{2} \\
& w_{q}(n+1)=w_{q}(n)+\Delta w_{q} \\
& m_{q i}(n+1)=m_{q i}(n)+\Delta m_{q i} \\
& \sigma_{q i}(n+1)=\sigma_{q i}(n)+\Delta \sigma_{q i} \\
& \Delta w_{q}=\eta_{q}\left(\theta_{m}-\theta_{a}\right)^{2} \exp \left[-\sum_{i}^{I} \frac{\left(f_{N N}(i)-m_{q i}(n)\right)^{2}}{\sigma_{q i}^{2}(n)}\right] \\
& \Delta m_{q i}=\eta_{m} 2 \exp \left[-\sum_{i}^{I} \frac{\left(f_{N N}(i)-m_{q i}(n)\right)^{2}}{\sigma_{q i}^{2}(n)}\right] \frac{\left(f_{N N}(i)-m_{q i}(n)\right)^{2}}{\sigma_{q i}^{2}(n)}\left(\theta_{m}-\theta_{a}\right) w_{q}(n)
\end{aligned}
$$




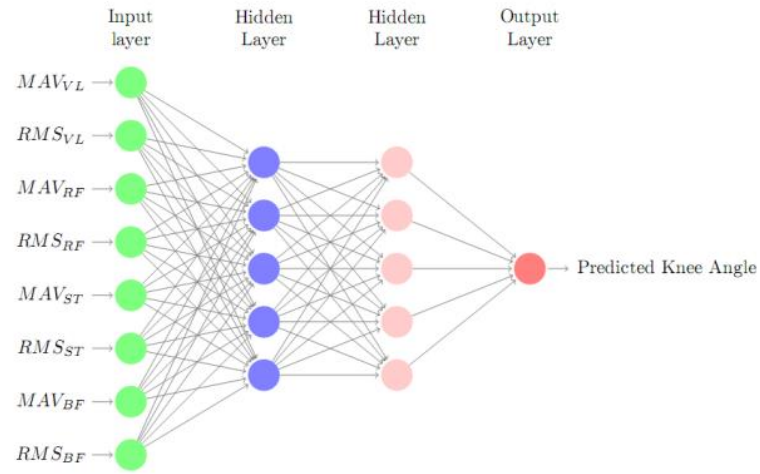

Fig. 3. Multi layer neural network used for predicting the knee angle

$\Delta \sigma_{q i}=\eta_{\sigma} \exp \left[-\sum_{i}^{I} \frac{\left(f_{N N}(i)-m_{q i}(n)\right)^{2}}{\sigma_{q i}^{2}(n)}\right] \frac{\left(f_{N N}(i)-m_{q i}(n)\right)^{2}}{\sigma_{q i}^{3}(n)}\left(\theta_{m}-\theta_{a}\right) w_{q}(n)$

Where $\eta_{q}, \eta_{m}$ and $\eta_{\sigma}$ are the learning constant, $\theta_{m}$ is the knee angle predicted and $\theta_{a}$ is the actual knee angle recorded by goniometer.

\section{Results}

Subjects were asked to perform daily life activities like normal walking, stand to sit in a squat and squat to stand operation, standing up and sitting on a chair and ascending and descending of stairs. sEMG signals were recorded from VL, STEN, BF and GMED muscles of lower limb. Knee angle was measured from the potentiometer based goniometer and NI 6009.

The recorded data was processed and time domain features MAV and RMS were obtained. The BPN was first trained using the $60 \%$ of the data and knee angle was predicted. The predicted knee angle and actual knee angle for Subject 1 are plotted in Fig 4. The accuracy of the predicted knee angle using the proposed algorithm is 92.4\%. The predicted knee angle could be used to control an exoskeleton or other orthotic or prosthetic device.

\section{Conclusion}

The present study proposed an algorithm for predicting knee angle from sEMG signals recorded from four muscles of human lower limb. The proposed algorithm accurately predicts the knee angle and could be used for controlling the exoskeleton myoelectrically. 


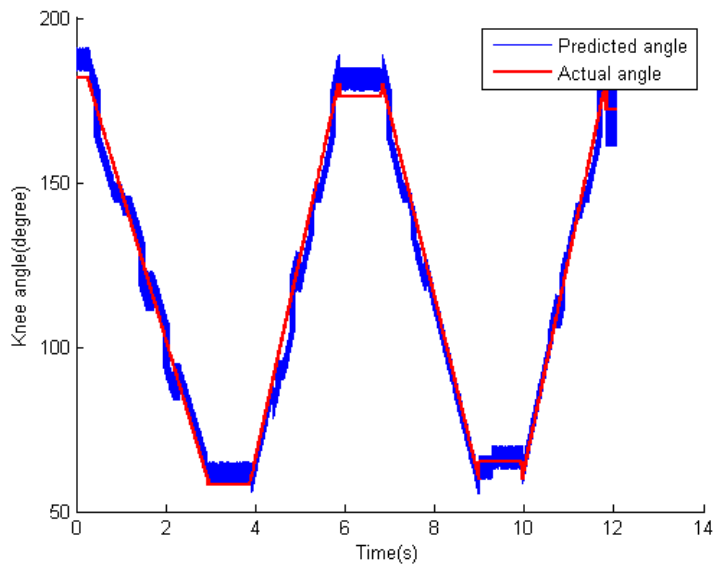

Fig. 4. The Predicted and measured knee angle

\section{References}

1. Yagn N. Apparatus for facilitating walking, running and jumping, 1890. US Patent 420, 179. URL: https://www.google.com/patents/ US420179.

2. Fickand B, Markinson J. Final Report On HARDIMAN-I Prototype For Machine Augmentation Of Human Strength And Enduance, Tech.Rep., General Electric Company, Schenectady, New York, 1971; 12345.

3. Moore J. L.A.N. Laboratory. PITMAN, a Powered Exoskeletal Suit for the Infantryman, Los Alamos National Laboratory, 1986. URL: http://books.google.co.in/books? id=stisGwAACAAJ.

4. Walsh C, Endo K, Herr H. A quasi -passive leg exoskeleton for load-carrying augmentation, International Journal of Humanoid Robotics, 2007; 4: 487-506.

5. Zoss A, Kazerooni H, Chu A. Biomechanical design of the Berkeley lower extremity exoskeleton (BLEEX), Mechatronics. A SME Transactions on IEEE, 2006; 11: 128-138.

6. Guizzo E, Goldstein H. The rise of the body bots [robotic exoskeletons], Spectrum, IEEE, 2005; 42: 50-56.

7. Lee S, Sankai Y. Power assist control for walking aid with HAL-3 based on EMG and impedance adjustment a round knee joint, 2002; 2: 1499-1504.

8. SENIAM group. Recommendations for sensor locations on individual muscles, 2013. URL: http://seniam.org/sensor_location.htm.

9. Stegeman D, Hermens H. Standards for surface electromyography: The European project surface emg for non-invasive assessment of muscles (seniam), 2007.

10. Olney SJ, Winter DA. Predictions of knee and ankle moments of force in walking from emg and kinematic data, Journal of biomechanics, 1985; 18: 9-20. 